'Departamento de Medicina Interna, Servicio de

Gastroenterología, Hospital Clínico Universidad de Chile. Santiago, Chile.

2Programa Enfermedad Inflamatoria Intestinal, Servicio de Gastroenterología, Clínica Las Condes. Santiago, Chile.

Los autores declaran no tener financiamiento ni conflictos de interés. Trabajo no recibió financiamiento.

Recibido el 1 de agosto de 2019, aceptado el 2 de enero de 2020 .

Correspondencia a: Edith Pérez de Arce Oñate Santos Dumont 999, Independencia. Santiago, Chile. eperezdearce@hcuch.cl

\section{Biomarcadores en enfermedad inflamatoria intestinal: ¿sabe cómo utilizarlos?}

\author{
EDITH PÉREZ DE ARCE ${ }^{1}$, ROCÍO SEDANO ${ }^{1}$, RODRIGO QUERA ${ }^{2}$
}

\section{Biomarkers in inflammatory bowel disease}

Biomarkers in inflammatory bowel disease are an essential tool in clinical practice. They allow a non-invasive evaluation of patients and thus guide decision-making at different stages of the disease, including diagnostic suspicion, severity assessment, relapse prediction, and treatment response. Although biomarkers in blood such as erythrocyte sedimentation rate and $C$-reactive protein, are the most commonly used biomarkers, because their low cost and accessibility, they lack specificity. Currently, fecal biomarkers offer greater reliability, applicability, and specificity. Fecal calprotectin is the most commonly used marker. This review discusses the advantages and disadvantages of biomarkers in inflammatory bowel disease, as well as their clinical applications and new biomarkers currently under research.

(Rev Med Chile 2020; 148: 362-370)

Key words: Biomarkers; Inflammatory Bowel Diseases; Crohn Disease; Colitis, Ulcerative.

\section{E} 1 uso de escalas clínicas de síntomas es una práctica habitual en la evaluación de pacientes con enfermedad inflamatoria intestinal $(\text { EII })^{1-3}$. Sin embargo, la aparición de síntomas es tardía y dista de ser un fiel indicador del nivel de inflamación. Si bien el estudio endoscópico continúa siendo el estándar en la evaluación de la actividad inflamatoria, es un método invasivo, de alto costo y poco accesible. Es aquí donde tienen un rol los biomarcadores. Un biomarcador se define como "una característica que se puede medir de manera objetiva y es evaluada como un indicador de un proceso biológico normal, un proceso patogénico o como la respuesta farmacológica a una intervención" ideal" en EII debe reunir una serie de condiciones para ser confiable: ser sensible a la inflamación en cualquier sitio del tubo digestivo, específico para la enfermedad, que cuantifique su extensión y gravedad, fácil de realizar, simple interpretación, mínimamente invasivo, económico, de rápido procesamiento y de resultados reproducibles ${ }^{5,6}$. En EII, los biomarcadores nos ayudan frente a la sospecha diagnóstica, la evaluación de su gravedad, la predicción de recaída y la respuesta al tratamiento. En la actualidad, los biomarcadores en sangre y heces utilizados tienen una serie de limitaciones que deben considerarse al interpretar sus resultados.

Este artículo tiene como objetivo revisar la evidencia actual de los biomarcadores en EII, proponer algunas recomendaciones para su uso racional y revisar algunos biomarcadores que se encuentran en investigación.

\section{Biomarcadores en sangre}

Son los más utilizados dado su fácil acceso y procesamiento, y bajo costo.

\section{Velocidad de hemosedimentación (VHS)}

La VHS cuantifica la velocidad con la que los glóbulos rojos sedimentan en un tubo capilar. La presencia de reactantes de fase aguda aceleran este proceso por lo que el aumento de la VHS refleja inflamación. La edad, anemia, tabaco y algunos 
fármacos (salicilatos) pueden afectar sus valores. Es altamente inespecífica, puesto que su elevación ocurre en una serie de condiciones inflamatorias. Su descenso tarda días o semanas después de la resolución del cuadro agudo ${ }^{7}$, lo que limita su uso en EII, ya que no es capaz de pesquisar a tiempo los cambios en la actividad inflamatoria ${ }^{8}$.

\section{Proteina C Reactiva (PCR)}

La PCR es un reactante de fase aguda producida en los hepatocitos durante estados de inflamación, por estimulación de citoquinas proinflamatorias donde destaca el factor de necrosis tumoral alfa $(\mathrm{TNF} \alpha)$ y las interleuquinas 1 y 6 . Tiene una vida media corta, de 19 horas, por lo que su elevación refleja actividad inflamatoria aguda, para luego descender rápidamente al resolverse el cuadro. Esta elevación es inespecífica, pudiendo aumentar frente a infecciones, enfermedades autoinmunes, trauma, neoplasias y otras condiciones. Existe una variabilidad interindividual en su producción que es dependiente de la edad, del índice de masa corporal (IMC), tabaco, fármacos (estatinas) y factores genéticos, donde algunos polimorfismos y haplotipos específicos pueden determinar su alta o baja expresión; es así como en $20 \%$ de la población, los niveles de PCR se mantienen bajos a pesar del estado inflamatorio activo".

Estudios clínicos sobre la PCR en el diagnóstico diferencial de síndrome de intestino irritable (SII) y EII han reportado una sensibilidad entre 70 y $100 \%$, dependiendo del punto de corte utilizado 9 . En una revisión sistemática, se encontró que pacientes con $\mathrm{PCR} \leq 5 \mathrm{mg} / \mathrm{L}$ tenían una probabilidad $\leq 1 \%$ de cursar con $\mathrm{EII}^{8}$. Existe poca evidencia sobre la utilidad de la PCR en detectar curación de la mucosa en pacientes con colitis ulcerosa (CU). Un estudio reportó que $49 \%$ de estos pacientes tenían PCR en rango normal, a pesar de presentar actividad inflamatoria en la colonoscopía $^{10}$. En enfermedad de Crohn (EC), la PCR también es producida por adipocitos mesentéricos, en respuesta a la inflamación local y a la translocación bacteriana a la grasa mesentérica ${ }^{11}$. A pesar de esta consideración, los resultados en EC son controversiales: estudios han mostrado que su aumento puede indicar enfermedad activa y correlacionarse con compromiso endoscópico e histológico ${ }^{12,13}$. Sin embargo, un metaanálisis reciente ${ }^{13}$ encontró una alta especificidad $(0,92)$, pero una baja sensibilidad $(0,49)$ de PCR para
EC con actividad endoscópica. En relación a la respuesta al tratamiento, estudios han reportado que pacientes con EC con PCR elevada al inicio de la terapia anti-TNF, tienen una mejor respuesta al fármaco ${ }^{14-16} \mathrm{y}$ su descenso durante el tratamiento predice una respuesta favorable a largo plazo $^{14}$. Por otra parte, en CU grave, la presencia de 3-8 deposiciones en $24 \mathrm{~h}$, asociado a una PCR $>45 \mathrm{mg} / \mathrm{L}$, tiene un valor predictivo positivo para necesidad de colectomía de $85 \%{ }^{17}$. No se ha demostrado utilidad de PCR en predicción de recaída ${ }^{18,19}$ y recurrencia postquirúrgica ${ }^{20}$, siendo su rendimiento inferior a calprotectina fecal (CF) (área bajo la curva (AUC) 0,70 vs 0,86 , respectivamente).

Por lo referido, la evidencia disponible no recomienda utilizar PCR y VHS aisladamente para la toma de decisiones en pacientes con EII.

\section{Biomarcadores fecales}

\section{Calprotectina fecal}

CF es una proteína de unión a calcio y zinc, miembro de la familia S100. Se encuentra predominantemente en neutrófilos, conformando $60 \%$ de las proteínas citosólicas, siendo liberada tras su activación ${ }^{21}$. Dentro de sus funciones se describe un rol antibacteriano directo, privando a los microorganismos de metales de transición, además de un efecto antiproliferativo e inmunomodulador $^{22}$. Está presente en varios fluidos corporales (plasma, orina y líquido sinovial) en proporción a la intensidad de la inflamación, sin embargo, su concentración en deposiciones es seis veces más alta que en el plasma y, dada su resistencia a la degradación enzimática, puede medirse hasta 7 días después de su liberación, lo que la hace un buen marcador de inflamación intestinal ${ }^{21}$. Si bien es sensible, no es específica, ya que puede elevarse en EII, cáncer de colon, diverticulitis, colitis infecciosas, colitis isquémica y enteropatía secundaria a antiinflamatorios no esteroidales (AINES), entre $\operatorname{otras}^{23}$ (Figura 1).

La toma de muestras fecales puede realizarse indistintamente en la mañana o en la tarde y conservarse hasta 3 días a temperatura ambiente, y no se recomienda después de la preparación para una colonoscopía o toma de biopsias ${ }^{24}$. Puede medirse por técnica de ELISA (ensayo por inmunoabsorción ligado a enzimas), que permite una medición cuantitativa (la más recomendada), o por ensayos de detección rápida (inmunocromatografía), que 
entregan resultados semicuantitativos o cualitati$\operatorname{vos}^{21}$. Debemos mencionar que existe variabilidad de los resultados de CF dependiente de los diferentes kits comerciales, por lo que se recomienda no comparar resultados obtenidos con diferentes kits en un mismo paciente ${ }^{24}$.

Un valor de $\mathrm{CF}<50 \mu \mathrm{g} / \mathrm{g}$ ha sido usado como valor de referencia normal en población general, sin embargo, esta cifra suele ser mayor en edades extremas ${ }^{21}$. En población pediátrica, el valor normal entre 1 y 3,9 años es CF $<77 \mu \mathrm{g} / \mathrm{g}$ y en $>4$ años se acepta el valor de referencia del adulto ${ }^{25}$. En $>65$ años se ha sugerido valores de corte de CF de $112 \mu \mathrm{g} / \mathrm{g}^{26} \mathrm{y}$ su ascenso se ha atribuido a cambios dietarios y de estilos de vida, cambios en las células inflamatorias de la mucosa gastrointestinal y en la inmunidad celular y humoral asociados a la edad ${ }^{21}$. Por otra parte, la CF no sería afectada durante el embarazo, por lo que es una herramienta útil en este escenario ${ }^{27}$.

\section{Calprotectina fecal en enfermedad inflamatoria intestinal}

La distinción entre enfermedad intestinal funcional y orgánica mediante CF ha sido objeto de varios estudios. Menees y cols. encontraron que pacientes con $\mathrm{CF} \leq 40 \mu \mathrm{g} / \mathrm{g}$ tienen una probabilidad $\leq 1 \%$ de padecer EII ${ }^{8}$. Un metaanálisis ${ }^{28}$ que evaluó la precisión diagnóstica de CF en EII reportó una sensibilidad de 0,95 , especificidad de 0,91 y AUC de 0,95 para el diagnóstico de EII, con mayor precisión diagnóstica para un punto de corte de $100 \mu \mathrm{g} / \mathrm{g}$ que $50 \mu \mathrm{g} / \mathrm{g}$. El retardo en el diagnóstico por un falso negativo ocurrió entre 6 y $8 \%$ de los pacientes ${ }^{29}$.
Recientemente, se ha sugerido el uso de CF en el estudio de pacientes con diarrea crónica con un valor de corte de $50 \mu \mathrm{g} / \mathrm{g}$ para optimizar la sensibilidad para $\mathrm{EII}^{30}$. Por otra parte, otros señalan tres rangos de $\mathrm{CF}$ para la orientación diagnóstica ${ }^{31}$ : pacientes con CF $>250 \mu \mathrm{g} / \mathrm{g}$ se beneficiarán de un estudio endoscópico precoz; valores intermedios (100-250 $\mu \mathrm{g} / \mathrm{g}$ ) deberán ser interpretados con precaución, considerando repetir el test $y$, junto a una historia clínica compatible, decidir qué pacientes necesitarán estudio endoscópico; CF < $100 \mu \mathrm{g} / \mathrm{g}$ no requerirán un estudio invasivo inmediato, debiéndose plantear diagnósticos diferenciales no inflamatorios. Puntos de corte más bajos aumentan la sensibilidad del test para EII, sin embargo, también aumenta el número de colonoscopias innecesarias por falsos positivos. Puntos de corte más altos aumentarán la especificidad, pero también la proporción de pacientes no diagnosticados.

Al contrario de lo que ocurre con la evaluación clínica en EII, CF tiene una fuerte correlación con la actividad endoscópica ${ }^{32-34}$ con una alta sensibilidad y especificidad global (88\% y $73 \%)$, pero con mejor rendimiento para CU que para EC (sensibilidad $88 \%$ vs $87 \%$ y especificidad $87 \%$ vs $67 \%$, respectivamente) ${ }^{13}$. A nivel nacional, $82,1 \%$ de los pacientes con EII endoscópicamente activa (Índice Mayo endoscópico 2-3) tenía CF $\geq 250$ $\mu \mathrm{g} / \mathrm{g}^{35}$. Un metaanálisis de 13 estudios prospectivos (744 pacientes con CU y 727 con EC) evaluó la precisión de CF para distinguir pacientes con EII en remisión de aquellos con enfermedad endoscópica activa ${ }^{36}$. Se encontró un AUC de 0,89 para distinguir EII activa de inactiva, con mejor rendimiento para $\mathrm{CU}$ (AUC 0,93 vs 0,88 para

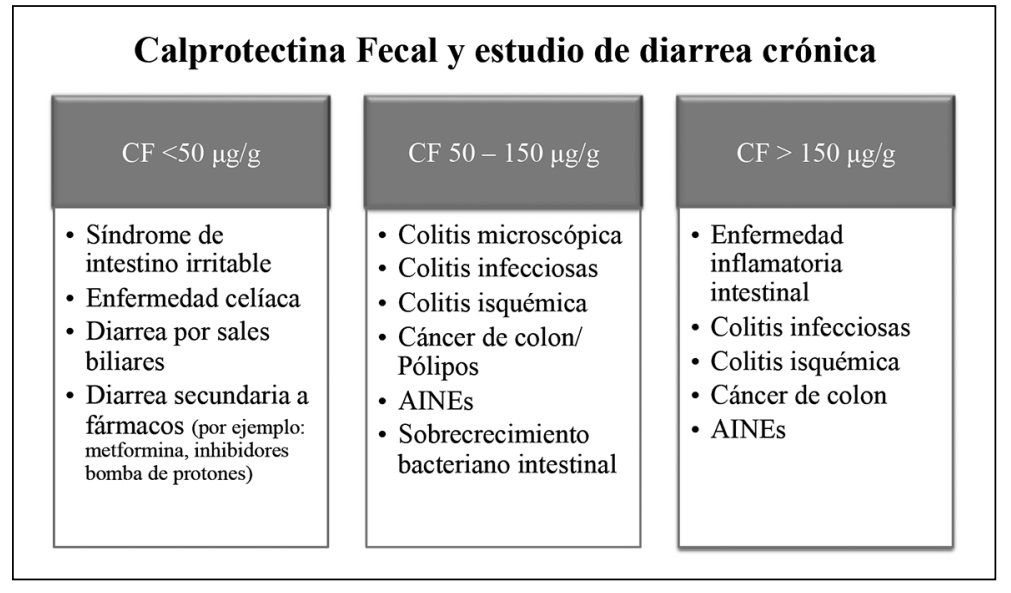

Figura 1. Orientación diagnóstica según valores de calprotectina fecal (CF) durante el estudio de diarrea crónica. 
EC). Un punto de corte de CF $250 \mu \mathrm{g} / \mathrm{g}$ mostró mejores resultados con una sensibilidad de $0,80 \mathrm{y}$ una especificidad de 0,82 .

La utilidad de CF en predecir el curso clínico de pacientes con CU aguda grave también ha sido investigada ${ }^{37,38}$. Un estudio reciente mostró que $\mathrm{CF}>1.000 \mu \mathrm{g} / \mathrm{g}$ al día 3 de terapia intensiva con esteroides endovenosos, fue predictor de no respuesta a esteroides y de necesidad de terapia de rescate o colectomía ${ }^{38}$. No se ha encontrado una relación significativa entre los niveles de CF y la extensión de la enfermedad en CU, a diferencia de lo visto entre CF y la gravedad de las lesiones endoscópicas ${ }^{39}$.

En EC, CF ha demostrado ser confiable cuando el compromiso es colónico o ileocolónico En EC exclusivamente ileal, reportes que han usado colonoscopía han señalado que su precisión diagnóstica es menor ${ }^{40}$. Sin embargo, estudios recientes con evaluación de intestino delgado mediante enteroscopía con balón ${ }^{41}$, resonancia magnética ${ }^{42}$ o cápsula endoscópica ${ }^{43}$, sugieren que $\mathrm{CF}$ es un buen biomarcador para compromiso ileal, aunque un poco menor que para compromiso colónico.

En relación a predicción de recaída, estudios observacionales han mostrado que el aumento de CF en CU inactiva puede predecir una recaída hasta 3 meses antes de la aparición de síntomas ${ }^{44,45}$, y que mediciones consecutivas en pacientes en remisión clínica son mucho más útiles que mediciones aisladas. Es así como valores normales de CF pueden correlacionarse con remisión histológica en $\mathrm{CU}^{46}$. Se recomienda controlar CF cada 3 a 4 meses durante el tratamiento de inducción o cambios de tratamiento, y en pacientes en remisión sostenida con evidencia de curación endoscópica, el control puede ser semestral ${ }^{24}$.

La disminución de CF puede ser un buen predictor de los cambios endoscópicos e histológicos en respuesta a la terapia de inducción y remisión clínica $^{47,48}$. Valores bajos de CF después de la inducción con anti-TNF puede predecir remisión sostenida durante 1 año, con una sensibilidad y especificidad de 80 y $85 \%$, respectivamente ${ }^{49}$. En relación a recurrencia postquirúrgica, un punto de corte de $50 \mu \mathrm{g} / \mathrm{g}$ puede predecir recurrencia endoscópica con una sensibilidad de $84,4 \%$ y una especificidad de $44,4 \%{ }^{50}$. De manera similar, un reciente metaanálisis que incluyó 613 pacientes con EC encontró una alta sensibilidad $(0,82)$ y baja especificidad $(0,61)$ para CF en predicción de recurrencia postquirúrgica ${ }^{51}$. En la predicción de reservoritis, la medición de CF demostró sensibilidad de $100 \%$ y especificidad de $84 \%$, con un punto de corte de $56 \mu \mathrm{g} / \mathrm{g}^{52}$, mostrando ser una herramienta útil para dirigir una evaluación endoscópica precoz $z^{53}$.

Durante los últimos años, la importancia clínica de los biomarcadores en EII ha ido en aumento. En 2015, el consenso de expertos STRIDE (Selecting Therapeutic Targets in Inflammatory Bowel Disease) $)^{33}$ acuñó la estrategia "Treat to Target" (T2T), que cambió las metas de tratamiento de la CU de prevención de complicaciones (hospitalizaciones, colectomías, displasia/cáncer) por la monitorización de mediciones objetivas de la actividad de la enfermedad (sangrado rectal, inflamación endoscópica de la mucosa, etc.), con la finalidad de cambiar su curso y mejorar la calidad de vida de los pacientes. En esta estrategia, el uso de biomarcadores se consideró como medida secundaria o auxiliar, sin embargo, la evidencia actual apoya el uso de métodos no invasivos como "objetivos de tratamiento" por su capacidad de predecir recaídas y respuesta a la terapia de inducción, sustituyendo, incluso, a la evaluación endoscópica ${ }^{9,54}$. En CU, una CF $<100 \mu \mathrm{g} / \mathrm{g}$ se podría considerar un objetivo de tratamiento al indicar una baja actividad inflamatoria ${ }^{54}$, y el mismo valor de corte ha sido sugerido como predictor de recurrencia endoscópica postquirúrgica en pacientes con $\mathrm{EC}^{20}$. "CALM", un estudio prospectivo, con distribución aleatoria de pacientes, en dos grupos, uno "Control Estrecho", que incorporaba monitorización con clínica y biomarcadores y otro grupo "Control Clínico"que controlaba solo con parámetros clínicos, en pacientes con EC moderada-grave ${ }^{55}$ demostró que el grupo de pacientes con un algoritmo de intensificación de terapia basado en un "Control Estrecho" de monitorización de síntomas (CDAI) y biomarcadores (PCR y CF), con una estrategia T2T fue más exitosa que el grupo "Manejo Clínico" basado solo en síntomas (CDAI). La estrategia "Control Estrecho" logró mayores tasas de remisión profunda (36,9\% vs $23 \%$ ) y biológica $(29,5 \%$ vs $15,6 \%)$, mayor curación de la mucosa con ausencia de úlceras profundas ( $46 \%$ vs $30 \%$ ) y mejores tasas de remisión clínica libre de esteroides, comparado con el grupo "Manejo Clínico".

En resumen, CF ha demostrado ser un bio- 
marcador confiable en EII con utilidad durante todo el curso de la enfermedad, siendo actualmente un objetivo terapéutico. Sus principales limitaciones están relacionadas con su costo y los múltiples kits utilizados para su procesamiento que pueden variar su interpretación.

\section{Otros biomarcadores fecales}

Lactoferrina y S100A12 tienen un menor uso en EII. Lactoferrina es una proteína de unión a fierro secretada por células del epitelio glandular y gránulos secundarios de los neutrófilos de la mucosa intestinal. Su liberación es proporcional al grado de inflamación de la mucosa, siendo un marcador sensible y específico. Es estable en deposiciones entre 2 a 5 días $^{6}$, aunque menos que CF. $\mathrm{Su}$ rol en diferenciar EII de enfermedad funcional ha mostrado resultados controversiales ${ }^{56,57}$, sin embargo, frente a una crisis, se ha documentado su elevación ${ }^{58}$. S100A12 representa el 5\% de las proteínas citosólicas del neutrófilo, y puede ser inducible en monocitos y células T. Similar a $\mathrm{CF}$, su liberación se produce frente al daño de la mucosa ${ }^{6}$. Si bien existen reportes sobre su utilidad en diferenciar SII de EII (sensibilidad: 86\%, especificidad: $96 \%)^{59}$, y como predictor de respuesta a agentes anti-TNF en pacientes con $\mathrm{EC}^{60}$, su medición no se encuentra estandarizada y los puntos de corte no han sido establecidos, por lo que no se recomienda su uso rutinario.

\section{Nuevos biomarcadores en EII}

\section{Test inmunológico de sangre oculta en deposiciones (TSODi)}

También conocido como FIT (Faecal Immunochemical Test), es un biomarcador fecal utilizado en el tamizaje de cáncer colorrectal, que cuantifica la concentración de hemoglobina en deposiciones mediante un anticuerpo específico. En CU, un valor de $<100 \mathrm{ng} / \mathrm{ml}$ es un buen predictor de curación de la mucosa (sensibilidad: $92 \%$, especificidad: $71 \%)^{61}$. Además, la especificidad mejora a $>90 \%$ cuando el TSODi es usado en combinación con $\mathrm{CF}^{62,63}$, aumentando la probabilidad de predecir riesgo de recaída ${ }^{64}$. Sin embargo, en EC, TSODi sería menos sensible a lesiones del intestino delgado en comparación con $\mathrm{CF}^{38,44,65}$. Las principales ventajas del TSDOi sobre CF son su bajo costo, facilidad en la toma de muestra y rápido procesamiento en sistemas automatizados. A pesar de los resultados prometedores, estudios deben aún confirmar su utilidad en EII.

\section{Otros biomarcadores en desarrollo}

El descubrimiento de genes de susceptibilidad para EII, del rol del microbioma intestinal, el desarrollo de la metabolómica, proteómica y adelantos en inmunología han permitido la identificación de nuevos potenciales biomarcadores. El objetivo es personalizar el manejo de estos pacientes mediante la caracterización de fenotipos moleculares más que fenotipos clínicos, que permitan predecir el riesgo de padecer la enfermedad, su comportamiento en el tiempo, predecir respuesta a fármacos y eventos adversos asociados. Esto es lo que conocemos como medicina de precisión ${ }^{66}$. Algunos de estos nuevos biomarcadores incluyen biomarcadores fecales, como el factor activante de células B (BAFF), M2-piruvato kinasa fecal, HMGB1 y NGAL fecal ${ }^{67}$ y el PROMOTHEUS ${ }^{\circledR}$ Monitr Crohn's Disease Test (Prometheus Laboratories Inc, San Diego, CA, USA) (Monitr) ${ }^{68}$. Este último es un novedoso examen en sangre periférica que identifica 13 biomarcadores asociados a vías de daño y reparación de mucosa. Un algoritmo recoge estas mediciones creando un "índice de curación de la mucosa"68. En la cohorte de validación, Monitr mostró 90\% de precisión con inflamación confirmada por ileocolonoscopía, (sensibilidad: $94 \%$, especificidad: 82\%). Además, demostró utilidad en EC con compromiso de intestino delgado aislado, con similar sensibilidad y especificidad para compromiso ileocolónico y colónico y, últimamente, en EC postoperatorio ${ }^{69}$. A pesar de estos resultados, se requieren publicaciones definitivas sobre este kit.

En cuento a biomarcadores serológicos, se encuentra el test Prometheus ${ }^{\circledR}$ IBD Serology 7 (Prometheus Laboratories Inc., San Diego, CA, USA), y su actualización, Prometheus ${ }^{\circledR}$ IBD sgi Diagnostic (Prometheus Laboratories Inc., San Diego, CA, USA $)^{70,71}$. Este último combina marcadores serológicos, genéticos y de inflamación, utilizando un algoritmo patentado de diagnóstico inteligente para tamizaje de EII y para diferenciar EC de CU. Sin embargo, debido a sus modestos resultados, se ha desestimado su uso en la práctica clínica.

Recientemente se ha descrito que el transcrip- 


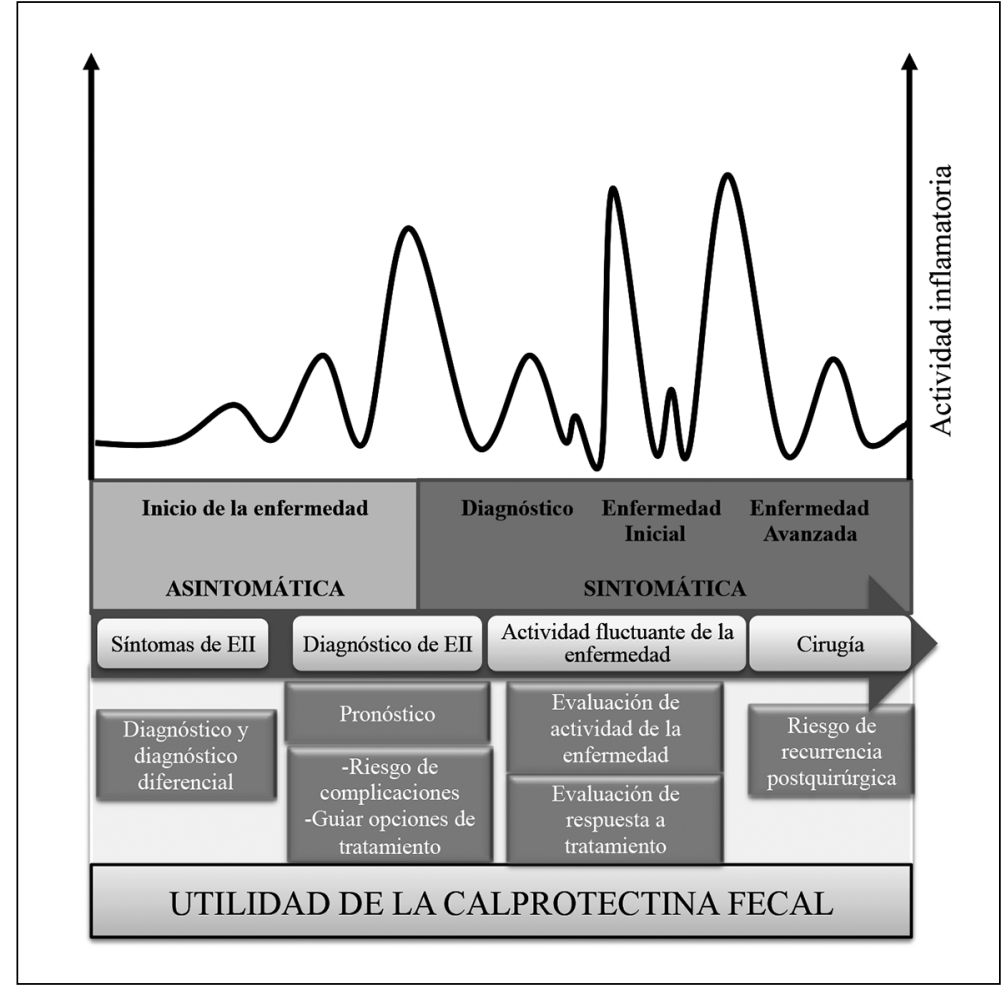

Figura 2. Utilidad de la calprotectina fecal durante la evolución de la Enfermedad Inflamatoria Intestinal. Adaptado de Rogler $\mathrm{G}$ et al. Curr Gastroenterol Rep (2015). (ref.73). toma de linfocitos T CD8+ permite diferenciar 2 subgrupos de pacientes con EII fenotípicamente distintos, siendo un prometedor marcador pronóstico, con una sensibilidad de $72,7 \%$ en EC y $100 \%$ en CU, y un valor predictivo negativo de $90,9 \%$ en EC y $100 \%$ en $\mathrm{CU}^{72}$.

\section{Conclusión}

El uso de biomarcadores ha facilitado el seguimiento de pacientes con EII, evitando la repetición de estudios invasivos. Algunos han mostrado utilidad en puntos específicos dentro del curso de la enfermedad y otros durante todas las etapas, como es el caso de CF (Figura 2 ) $^{65}$, cuya medición sistemática en la actualidad ha sido considerada como un objetivo de tratamiento. Aún estamos distantes de un biomarcador ideal, por lo que conocer las ventajas, desventajas y limitaciones de los actuales nos permitirá un uso racional acorde a las necesidades del paciente. El acelerado avance científico permitirá próximamente disponer de biomarcadores que permitan aplicar una medicina de precisión de calidad.

\section{Referencias}

1. Harvey RF, Bradshaw JM. A simple index of Crohn's-disease activity. Lancet 1980; 1 (8167): 514.

2. Best WR, Becktel JM, Singleton JW, Kern F. Development of a Crohn's disease activity index. National Cooperative Crohn's Disease Study. Gastroenterology 1976, 70 (3): 439.

3. Rutgeerts P, Sandborn WJ, Feagan BG, Reinisch W, Olson A, Johanns J, et al. Infliximab for induction and maintenance therapy for ulcerative colitis. $\mathrm{N}$ Engl $\mathrm{J}$ Med. 2005; 353 (23): 2462-76.

4. Biomarkers Definitions Working Group. Biomarkers and surrogate endpoints: preferred definitions and conceptual framework. Clin Pharmcol Ther 2001; 69 (3): 89-95.

5. Vermeire S, Van Assche G, Rutgeerts P. Laboratory markers in IBD: useful, magic, or unnecessary toys? Gut 2006; 55 (3): 426-31.

6. López RN, Leach ST, Lemberg DA, Duvoisin G, Gearry RB, Day AS. Fecal biomarkers in inflammatory bowel disease. J Gastroenterol Hepatol. 2017; 32 (3): 577-82.

7. Litao MK, Kamat D. Erythrocyte Sedimentation Rate 
and C-Reactive Protein: How Best to Use Them in Clinical Practice. Pediatr Ann. 2014; 43 (10): 417-20.

8. Menees SB, Powell C, Kurlander J, Goel A, Chei WD. A meta-analysis of the utility of C-reactive protein, erythrocyte sedimentation rate, fecal calprotectin, and fecal lactoferrin to exclude inflammatory bowel disease in adults with IBS. Am J Gastroenterol 2015; 110 (3): 444-54.

9. Ma C, Battat R, Parker CE, Khanna R, Jairath V, Feagan BG. Update on C-reactive protein and fecal calprotectin: are they accurate measures of disease activity in Crohn's disease? Expert Rev Gastroenterol Hepatol 2019; 13 (4): 319-30.

10. Solem CA, Loftus EV, Tremaine WJ, Harmsen WS, Zinsmeister AR, Sandborn WJ. Correlation of C-reactive protein with clinical, endoscopic, histologic, and radiographic activity in inflammatory bowel disease. Inflamm Bowel Dis. 2005; 11 (8): 707-12.

11. Peyrin-Biroulet L, González F, Dubuquoy L, Rousseaux C, Dubuquoy C, Decourcelle C, et al. Mesenteric fat as a source of $\mathrm{C}$ reactive protein and as a target for bacterial translocation in Crohn's disease. Gut. 2012; 61 (1): 7885.

12. Hosomi S, Yamagami H, Itani S, Yukawa T, Otani K, Nagami Y, et al. Sepsis markers soluble IL-2 receptor and soluble CD14 subtype as potential biomarkers for complete mucosal healing in patients with inflammatory bowel disease. J Crohn Colitis 2018; 12 (5): 87-95.

13. Mosli MH, Zou G, Garg SK, Feagan SG, MacDonald JK, Chande N, et al. C-reactive protein, fecal calprotectin, and stool lactoferrin for detection o endoscopic activity in symptomatic inflammatory bowel disease patients: A systematic review and meta-analysis. Am J Gastroenterol 2015; 110 (6): 802-19.

14. Reinisch W, Wang Y, Oddens BJ, Link R. C-reactive protein, an indicator for maintained response or remission to infliximab in patients with Crohn's disease: a post-hoc analysis from ACCENT I. Aliment Pharmacol Ther. 2012; 35 (5): 568-76.

15. Jürgens M, Mahachie John JM, Cleynen I, Schnitzier F, Fidder H, van Moerkercke W, et al. Levels of C-reactive protein are associated with response to infliximab therapy in patients with Crohn's disease. Clin Gastroenterol Hepatol. 2011; 9 (5): 421-7.

16. Sandborn WJ, Colombel JF, D’Haens G, Plevy SE, Panés J, Robinson AM, et al. Association of baseline C-reactive protein and prior anti-tumor necrosis factor therapy with need for weekly dosing during maintenance therapy with adalimumab in patients with moderate to severe Crohn's disease. Curr Med Res Opin. 2013; 29 (5): 483-93.
17. Travis SP, Farrant JM, Ricketts C, Nolan DJ, Mortensen NM, Kettlewell MG, et al. Predicting the outcome of severe ulcerative colitis. Gut 1996; 38 (6): 905-10.

18. Langhorst J, Boone J, Lauche R, Rueffer A, Dobos G. Fecal Lactoferrin, Calprotectin, PMN-elastase, CRP, and white blood cell count as indicators for mucosal healing and clinical course of disease in patients with mild to moderate ulcerative colitis: post hoc analysis of a prospective clinical trial. J Crohn Colitis 2016; 10 (7): 786-94.

19. Dabritz J, Langhorst J, Lügering A, Heidemann J, Mohr $\mathrm{M}$, Wittkowski $\mathrm{H}$, et al. Improving relapse prediction in inflammatory bowel disease by neutrophil-derived S100A12. Inflamm Bowel Dis 2013; 19 (6): 1130-8.

20. Wright EK, Kamm MA, De Cruz P, Hamilton AL, Ritchie KJ, Krejany EO, et al. Measurement of Fecal Calprotectin Improves Monitoring and Detection of Recurrence of Crohn's Disease After Surgery. Gastroenterology. 2015; 148 (5): 938-47.

21. Ayling R, Kok K. Fecal Calprotectin. Adv Clin Chem. 2018; 87: 161-90.

22. Sutherland AD, Gearry RB, Frizelle FA. Review of fecal biomarkers in inflammatory bowel disease. Dis Colon Rectum. 2008; 51 (8): 1283-91.

23. Brookes MJ, Whitehead S, Gaya DR, Hawthorne AB. Practical guidance on the use of faecal calprotectin. Frontline Gastroenterol 2018; 9 (2): 87-91.

24. Guardiola J, Lobatón T, Cerrillo E, Ferreiro-Iglesias R, Gisbert JP, Domenech E, et al. Recomendaciones del Grupo Español de Trabajo en Enfermedad de Crohn y Colitis Ulcerosa (GETECCU) sobre la utilidad de la determinación de calprotectina fecal en la enfermedad inflamatoria intestinal. Gastroenterol Hepatol 2018; 41 (8): 514-29.

25. Davidson F, Lock RJ. Paediatric reference ranges for faecal calprotectin: a UK study. Ann Clin Biochem 2017; 54 (2): 214-8.

26. Joshi S, Lewis SJ, Creanor S, Ayling RM. Age related faecal calprotectina, lactoferrin and tumour M2-PK concentrations in healthy volunteers. Ann Clin Biochem 2010; 47 (Pt 3): 259-63.

27. Julsgaard M, Hvas CL, Gearry RB, Vestergaard T, Fallingborg J, Svenningsen L, et al. Fecal calprotectin is not affected by pregnancy: clinical implications for the management of pregnant patients with inflammatory bowel disease. Inflamm Bowel Dis 2017; 23 (7): 1240-6.

28. Von Roon AC, Karamountzos L, Purkayastha S, Reese GE, Darzi AW, Teare JP, et al. Diagnostic precision of fecal calprotectin for inflammatory bowel disease and colorectal malignancy. Am J Gastroenterol 2007; 102 (4): 803-13. 
29. van Rheenen PF, Van de Vijver E y Fidler V. Faecal calprotectin for screening of patients with suspected inflammatory bowel disease: Diagnostic meta-analysis. BMJ 2010; 341 (7765): c3369.

30. Smalley W, Falck-Ytter C, Carrasco-Labra A, Lytvyn L, Falck Ytter Y. American Gastroenterological Association Guideline on Mild-to-Moderate Ulcerative Colitis on the Laboratory Evaluation of Functional Diarrhea and Diarrhea-Predominant Irritable Bowel Syndrome in Adults (IBS-D). Gastroenterology 2019 Jul 11. [Epub ahead of print].

31. Reenaers C, Bossuyt P, Hindryckc P, Vanpoucke H, Cremer A, Baert F. Expert opinion for use of faecal calprotectin in diagnosis and monitoring of inflammatory bowel disease in daily clinical practice. United European Gastroenterol J 2018; 6 (8): 1117-25.

32. D'Haens G, Ferrante M, Vermeire S, Baert F, Noman $\mathrm{M}$, Moortgat L, et al. Fecal calprotectin is a surrogate marker for endoscopic lesions in inflammatory bowel disease. Inflamm Bowel Dis 2012; 18 (12): 2218-24.

33. Peyrin-Biroulet L, Sandborn W, Sands BE, Reinisch W, Bemelman W, D`Haens G, et al. Selecting Therapeutic Targets in Inflammatory Bowel Disease (STRIDE): Determining therapeutic goals for treat-to-target. Am J Gastroenterol 2015; 110 (9): 1324-38.

34. Sipponen T, Savilahti E, Kolho KL, Nuutinen H, Turunen U, Färkkilä M. Crohn's disease activity assessed by fecal calprotectin and lactoferrin: Correlation with Crohn's disease activity index and endoscopic findings. Inflamm Bowel Dis 2008; 14 (1): 40-6.

35. Fluxá D, Simian D, Flores L, Ibáñez P, Lubascher J, Figueroa C, et al. Clinical, endoscopic and histological correlation and measures of association in ulcerative colitis. J Dig Dis 2017; 18 (11): 634-41.

36. Lin JF, Chen JM, Zuo JH, Yu A, Xiao ZJ, Deng FH, Nie B, Jiang B. Meta-analysis: Fecal calprotectin for assessment of inflammatory bowel disease activity. Inflamm Bowel Dis. 2014; 20 (8): 1407-15.

37. Ho GT, Lee HM, Brydon G, Ting T, Hare N, Drummond $\mathrm{H}$, et al. Fecal calprotectin predicts the clinical course of acute severe ulcerative colitis. Am J Gastroenterol 2009; 104 (3): 673-8.

38. Jain S, Kedia S, Bopanna S, Sachdev V, Sahni P, Dash NR, et al. Faecal Calprotectin and UCEIS Predict Shortterm Outcomes in Acute Severe Colitis: Prospective Cohort Study. J Crohns Colitis 2017 27; 11 (11): 1309-16.

39. Røseth AG, Aadland E, Jahnsen J, Raknerud N. Assessment of disease activity in ulcerative colitis by faecal calprotectin, a novel granulocyte marker protein. Digestion. 1997; 58 (2): 176-80.

40. Stawczyk-Eder K, Eder P, Lykowska-Szuber L, Kre-
la-Kazmierczak I, Klimczak K, Szymczak A, et al. Is faecal calprotectin equally useful in all Crohn's disease loca-tions? A prospective, comparative study. Arch Med Sci. 2015; 11 (2): 353-61.

41. Inokuchi $\mathrm{T}$, Kato J, Hiraoka $\mathrm{S}$, Takashima $\mathrm{S}$, Nakarai A, Takei D, et al. Fecal immunochemical test versus fecal calprotectin for prediction of mucosal healing in Crohn's disease. Inflamm Bowel Dis 2016; 22 (5): 1078-85.

42. Cerrillo E, Beltrán B, Pous S, Echarri A, Gallego JC, Iborra M, et al. Fecal calprotectin in ileal Crohn's disease: Relationship with magnetic resonance enterography and a pathology score.Inflamm Bowel Dis. 2015; 21 (7): 1572-9.

43. Aggarwal V, Day AS, Connor S, Leach ST, Brown G, Singh R, et al. Role of capsule endoscopy and fecal biomarkers in small-bowel Crohn's disease to assess remission and predict relapse. Gastrointest Endosc. 2017; 86 (6): 1070-8.

44. De Vos M, Louis EJ, Jahnsen J, Vandervoort JG, Noman $\mathrm{M}$, Dewit $\mathrm{O}$, et al. Consecutive fecal calprotectin measurements to predict relapse in patients with ulcerative colitis receiving infliximab maintenance therapy. Inflamm Bowel Dis 2013; 19 (10): 2111-7.

45. Molander P, Färkkilä M, Ristimäki A, Salminen K, Kemppainen $\mathrm{H}$, Blomster $\mathrm{T}$, et al. Does fecal calprotectin predict short-term relapse after stopping TNF -blocking agents in inflammatory bowel disease patients in deep remission? J Crohn Colitis 2015; 9 (1): 33-40.

46. Kato J, Yoshida T, Hiraoka S. Prediction of treatment outcome and relapse in inflammatory bowel disease. Expert Rev Clin Immunol. 2019; 15 (6): 667-77.

47. Sandborn WJ, Panés J, Zhang H, Yu D, Niezychowski W, Su C. Correlation between concentrations of fecal calprotectin and outcomes of patients with ulcerative colitis in a phase 2 trial. Gastroenterology 2016; 150 (1): 96-102.

48. Reinisch W, Bressler B, Curtis R, Parikh A, Yang H, Rosario M, et al. Fecal calprotectin responses following induction therapy with vedolizumab in moderate to severe ulcerative colitis. A post hoc analysis of GEMINI 1. Inflamm Bowel Dis 2019; 25 (4): 803-10.

49. Gaudi L, Marzo M, Andrisani G, Felice C, Pugliese D, Mocci G, et al. Faecal calprotectin assay after induction with anti-tumour necrosis factor agents in inflammatory bowel disease: prediction of clinical response and mucosal healing at one year. Dig Liver Dis 2014; 46 (11): 974-9.

50. Mowat C, Arnott I, Cahill A, Smith M, Ahmad T, Subramanian S, et al. Mercaptopurine versus placebo to prevent recurrence of Crohn's disease after surgical 
resection (TOPPIC): a multicentre, double-blind, randomised controlled trial. Lancet Gastroenterol Hepatol. 2016; 1 (4): 273-82.

51. Qiu Y, Mao R, Chen BL, He Y, Zeng ZR, Xue L, et al. Fecal calprotectin for evaluating postoperative recurrence of Crohn's disease: a meta-analysis of prospective studies. Inflamm Bowel Dis. 2015; 21 (2): 315-22.

52. Yamamoto T, Shimoyama T, Bamba T, Matsumoto K. Consecutive Monitoring of Fecal Calprotectin Calprotectin and Lactoferrin for the Early Diagnosis and Prediction of Pouchitis after Restorative Proctocolectomy for Ulcerative Colitis. Am J Gastroenterol 2015; 110 (6): 881-7.

53. Schoepfer A, Reinish W. Serial Fecal Calprotectin and Lactoferrin Measurements for Early Diagnosis of Pouchitis After Proctocolectomy for Ulcerative Colitis: Is Pouchoscopy No Longer Needed? Am J Gastroenterol 2015; 110 (6): 888-90.

54. Ungaro R, Colombel JF, Lissoos T, Peyrin-Biroulet L. A Treat-to-Target Update in Ulcerative Colitis: A Systematic Review. Am J Gastroenterol 2019; 114 (6): 874-83.

55. Colombel JF, Panaccione R, Bossuyt P, Lukas M, Baert $\mathrm{F}, \mathrm{Va}$ ásek T, et al. Effect of tight control management on Crohn's disease (CALM): a multicentre, randomised, controlled phase 3 trial. Lancet 2018; 390 (10114): 277989.

56. Sherwood RA. Faecal markers of gastrointestinal inflammation. J. Clin. Pathol. 2012; 65 (11): 981-5.

57. Silberer H, Küppers B, Mickisch O, Baniewicz W, Drescher M, Traber M, et al. Fecal leukocyte proteins in inflammatory bowel disease and irritable bowel syndrome. Clin Lab 2005; 51 (3-4): 117-26.

58. Walker TR, Land ML, Kartashov A, Saslowsky TM, Lyerly DM, Bonne JH, et al. Fecal lactoferrin is a sensitive and specific marker of disease activity in children and young adults with inflammatory bowel disease. J. Pediatr. Gastroenterol. Nutr. 2007; 44 (4): 414-22.

59. Kaiser T, Langhorst J, Wittkowski H, Becker K, Friedrich AW, Rueffer A, et al. Faecal S100A12 as a noninvasive marker distinguishing inflammatory bowel disease from irritable bowel syndrome. Gut. 2007; 56 (12): 1706-13.

60. Boschetti G, Garnerro P, Moussata D, Cuerq C, Préaudat C, Duclaux-Loras R, et al. Accuracies of serum and fecal S100 proteins (calprotectin and calgranulin C) to predict the response to TNF antagonists in patients with Crohn's disease. Inflamm. Bowel. Dis. 2015; 21 (2): 3316.

61. Nakarai A, Kato J, Hiraoka S, Kuriyama M, Akita M, Hirakawa T, Okada H, Yamamoto K. Evaluation of mucosal healing of ulcerative colitis by a quantitative fecal immunochemical test. Am J Gastroenterol 2013;
108 (1): 83-9.

62. Takashima S, Kato J, Hiraoka S, Nakarai A, Takei D, Inokuchi $\mathrm{T}$, et al. Evaluation of Mucosal Healing in Ulcerative Colitis by Fecal Calprotectin Vs. Fecal Immunochemical Test. Am J Gastroenterol 2015; 110 (6): 873-80.

63. Shi HY, Chan FKL, Chan AWH, Higashimori A, Kyaw M, Ching JYL, et al. Accuracy of Faecal Immunochemical Test to Predict Endoscopic and Histological Healing in Ulcerative Colitis: A Prospective Study Based on Validated Histological Scores. J Crohns Colitis 2017; 11 (9): 1071-7.

64. Nakarai A, Hiraoka S, Takahashi S, Inaba T, Higashi R, Mizuno M, et al. Simultaneous Measurements of Faecal Calprotectin and the Faecal Immunochemical Test in Quiescent Ulcerative Colitis Patients Can Stratify Risk of Relapse. J Crohns Colitis 2018; 5; 12 (1): 71-6.

65. Kato J, Hiraoka S, Nakarai A, Takashima S, Inokuchi T, Ichinose M. Fecal immunochemical test as a biomarker for inflammatory bowel diseases: can it rival fecal calprotectin? Intest Res. 2016;14 (1): 5-14.

66. Boyapati RK, Kalla R, Satsangi J, Ho GT. Biomarkers in Search of Precision Medicine in IBD. Am J Gastroenterol 2016; 111 (12): 1682-90.

67. Ministro P, Martins D. Fecal biomarkers in inflammatory bowel disease: how, when and why? Expert Rev Gastroenterol Hepatol. 2017; 11 (4): 317-28.

68. Sandborn WJ, Abreu MT, Dubinsky MC. A Noninvasive Method to Assess Mucosal Healing in Patients With Crohn's Disease. Gastroenterol Hepatol (N Y). 2018; 14 (5 Suppl 2): 1-12.

69. Kelly OB, Silverberg MS, Dulai PS, Boland BS, Vermeire $S$, Laharie D, et al. Development and validation of a multi-marker serum test for the assessment of mucosal healing in Crohn's disease patients [WCOG abstract P2184]. Paper presented at: the World Congress of Gastroenterology at ACG2017; October 13-18, 2017; Orlando, FL.

70. Benor S, Rusell GH, Silver M, Yuan Q, Winter HS. Shortcomings of the inflammatory bowel disease Serology 7 panel. Pediatrics 2010; 125 (6): 1230-6.

71. Shirts B, von Roon AC, Tebo AE. The entire predictive value of the Prometheus IBD sgi diagnostic product may be due to the three least expensive and most available components. Am J Gastroenterol 2012; 107 (11): 17601.

72. Biasci D, Lee JC, Noor NM, Pombal DR, Hou M, Lewis $\mathrm{N}$, et al. A blood-based prognostic biomarker in IBD. Gut 2019; 68 (8): 1386-95.

73. Rogler G, Biedermann L. Clinical Utility of Biomarkers in IBD. Curr Gastroenterol Rep 2015; 17 (7): 26. 